\title{
The subchondral bone healing after fixation of an osteochondral talar defect is superior in comparison with microfracture
}

\author{
Mikel L. Reilingh ${ }^{1,2,3} \cdot$ Kaj T. A. Lambers ${ }^{1,2,3} \cdot$ Jari Dahmen $^{1,2,3} \cdot$ \\ Kim T. M. Opdam ${ }^{1,2,3}$ • Gino M. M. J. Kerkhoffs ${ }^{1,2,3}$
}

Received: 7 April 2017 / Accepted: 19 July 2017 / Published online: 27 July 2017

(C) The Author(s) 2017. This article is an open access publication

\begin{abstract}
Purpose Arthroscopic bone marrow stimulation (BMS) has been considered the primary surgical treatment for osteochondral defects (OCDs) of the talus. However, fixation has been considered as a good alternative. Recently, a new arthroscopic fixation technique was described: the lift, drill, fill and fix procedure (LDFF). The purpose of this study was to evaluate the clinical and radiological results between arthroscopic LDFF and arthroscopic BMS in primary fixable talar OCDs at 1-year follow-up.

Methods In a prospective comparative study, 14 patients were treated with arthroscopic BMS and 14 patients with arthroscopic LDFF. Pre- and postoperative clinical assessment included the American Orthopaedic Foot and Ankle Society (AOFAS) score and the numeric rating scales (NRSs) of pain at rest and running. Additionally, the level of the subchondral plate (flush or depressed) was analysed on the 1 year postoperative computed tomography scans.

Results No significant differences in the AOFAS and NRS pain at rest and running were found between both groups at 1-year follow-up. After LDFF the level of the
\end{abstract}

Mikel L. Reilingh

m.1.reilingh@amc.uva.nl

Gino M. M. J. Kerkhoffs

g.m.kerkhoffs@amc.uva.nl

1 Department of Orthopedic Surgery, Academic Medical Center, University of Amsterdam, Meibergdreef 9, 1105 AZ Amsterdam, The Netherlands

2 Academic Center for Evidence Based Sports Medicine (ACES), Meibergdreef 9, 1105 AZ Amsterdam, The Netherlands

3 Amsterdam Collaboration for Health and Safety in Sports (ACHSS), Meibergdreef 9, 1105 AZ Amsterdam, The Netherlands subchondral bone plate was flush in 10 patients and after BMS in three patients $(p=0.02)$.

Conclusion No clinical differences were found between arthroscopic LDFF and arthroscopic BMS in the treatment of talar OCDs at 1-year follow-up. However, the subchondral bone plate restores significantly superior after arthroscopic LDFF compared to arthroscopic BMS. It may therefore give less progression of ankle osteoarthritis in the future with a thus potential better long-term outcome.

Level of evidence III.

Keywords Ankle · Osteochondral defects · Arthroscopy · Microfracture - Bone marrow stimulation - Fixation - Lift, drill, fill and fix (LDFF)

\section{Introduction}

Osteochondral defects (OCDs) of the talus often have a severe impact on the quality of life of patients [25]. Currently, arthroscopic bone marrow stimulation (BMS) has been considered the primary surgical treatment for chronic OCDs up to $15 \mathrm{~mm}$. This preference is based on the ease of execution of the technique, the low complication rate and high success rates reported in the literature [30, 31]. However, BMS does not aim at the preservation of a hyaline cartilage layer, but rather promotes the formation of fibrocartilage which decreases in quality over time and shows inferior wear characteristics [12, 13, 23]. Furthermore, after debridement and bone marrow stimulation the subchondral bone plate is often irregular and depressed [19]. These factors might be the reason why progression of ankle osteoarthritis is seen in 33-34\% of the patients at long-term follow-up [4, 16, 24]. 
Recently, a new arthroscopic fixation technique for chronic primary talar OCDs was described: the lift, drill, fill and fix procedure (LDFF) [7]. The assumed theoretical advantages of this technique are the restoration of the subchondral bone plate and the preservation of hyaline cartilage. Promising clinical and radiological results were found in the first seven patients at 1-year follow-up. However, at present, no comparative study has been conducted between LDFF and BMS in primary fixable talar OCDs. Consequently, the aim of this study was to evaluate the clinical and radiological results between arthroscopic LDFF and arthroscopic BMS in primary fixable talar OCDs at 1-year follow-up.

\section{Materials and methods}

This study was approved by the local medical ethics committee at the University of Amsterdam with reference number MEC 08/326 and performed in accordance with the current ethical standards (Declaration of Helsinki).

The study included patients with a symptomatic fixable primary talar OCD with a diameter $>10 \mathrm{~mm}$ (in three dimensions) as measured on computed tomography (CT) scans. Fixable defects were defined as type II-IV, based on the Berndt and Harty classification [1]. Exclusion criteria were open physis of the distal tibia, ankle osteoarthritis grade II or III [26], concomitant OCD of the tibia, ankle fracture within 6 months before treatment of the OCD, surgical treatment of the index ankle performed within 1 year before treatment of the OCD, concomitant painful or disabling disease of the lower limb and rheumatoid arthritis.

\section{Population}

As of 2013, we have prospectively recorded all patients undergoing an arthroscopic LDFF procedure [7]. For the control group (arthroscopic BMS), we used data from a randomized controlled trial (RCT) investigating pulsed electromagnetic fields (PEMF) after arthroscopic debridement and BMS [20]. Both the PEMF treatment and the placebo group were included in the arthroscopic BMS control group of the present study, as neither functional nor radiological differences between the groups were found in the previous trial. Patients were retrospectively selected to the BMS control group if their lesion could be defined as a fixable defect.

\section{Operative technique}

\section{Arthroscopic LDFF}

All arthroscopic LDFF procedures were performed using a standardized technique by the senior author (GK) [7]. Anteromedial and anterolateral portals were created with the ankle in full dorsiflexion. The OCD was identified with a probe by moving the ankle in full plantar flexion. Subsequent to this, an osteochondral flap was created with use of a beaver knife and lifted with a chisel. The bone flake of the osteochondral fragment as well as the osteosclerotic area of the bed was drilled with the use of a K-wire and a shaver blade. Cancellous bone was harvested from the distal tibia and transported into the defect until there was sufficient substantial filling. Finally, the osteochondral flap was fixed with an absorbable bio-compression screw(s) (Arthrex Inc, Naples, USA) or/and a chondral dart(s) (Arthrex Inc, Naples, USA).

Postoperatively, a short-leg non-weight-bearing cast was applied for 4 weeks. After these 4 weeks, the foot was placed in a short-leg walking cast in neutral flexion position and neutral hindfoot position, with full weight bearing allowed. At 8 weeks postoperatively, the cast was removed. Physical therapy was prescribed to assist in functional recovery and extend to full weight bearing in approximately 2 weeks [7].

\section{Arthroscopic BMS}

All arthroscopic BMS procedures were performed using a standardized technique by the senior author (GK) [20]. Like in the LDFF technique, an anteromedial and an anterolateral portal was created. After identification of the OCD, all unstable bone and cartilage were removed with a curette and bone cutter shaver. This was followed by perforation with a microfracture awl, with intervals of approximately $3 \mathrm{~mm}$. At the end of the procedure, a pressure bandage was applied.

Postoperative management consisted of a protocol-based rehabilitation programme, guided by a physiotherapist. Partial (eggshell) weight bearing on crutches was allowed as tolerated and progressed to full weight bearing over a period of 6 weeks. During this 6-week period, active nonweight-bearing and partial weight-bearing sagittal range of motion exercises were encouraged [27].

\section{Outcome assessment}

Clinical outcome was assessed by means of numeric rating scales (NRSs) for pain (at rest and running) and the American Orthopaedic Foot and Ankle Society (AOFAS) ankle-hindfoot score $[5,6,8]$. These questionnaires were evaluated preoperatively and at 1 year postoperatively. The NRS is an 11-point scale, representing the spectrum of no pain (0 points) to the worst pain imaginable (10 points) [5]. The AOFAS is a 100-point score, with a subjective and an objective component, which devotes 40 points to pain, 50 to function and 10 to alignment $[6,8]$. 


\section{Imaging}

Computed tomography (CT) scans of the affected ankle were obtained preoperatively and at 1 year postoperatively. The scanning protocol involved "ultra-high-resolution" axial slices with an increment of $0.3 \mathrm{~mm}$ and a thickness of $0.6 \mathrm{~mm}$, and multi-planar coronal and sagittal reconstructions of $1.0 \mathrm{~mm}$ [20]. CT scanning has been proven to be accurate in the detection and follow-up of OCDs of the talus, regarding location and extent as well as healing of the defect [14, 20, 28, 32].

On the preoperative CT scans, we graded the talar OCDs according to the modified Berndt and Harty classification $[1,22]$ and evaluated the OCD size by measuring the largest diameter $(\mathrm{mm})$ in the anterior-posterior direction, medial-lateral direction and depth.

The level of the subchondral plate (flush or depressed) was analysed on the 1 year postoperative CT scans. Reilingh et al. [20] reported a good reliability in the measurements of the subchondral bone plate on CT scans. Furthermore, the union rate was evaluated on the postoperative $\mathrm{CT}$ scans after the LDFF procedure.

\section{Statistical analysis}

Statistical analyses were conducted with Statistical Packages for Social Sciences (SPSS 23.0 Inc, Chicago, IL, USA) software. Continues data are presented as means with standard deviations or as medians with interquartile ranges (IQRs), depending on their distribution (normal or skewed). Comparison of the clinical outcome between groups was performed by the Student's $t$ test on normal distribution and the Mann-Whitney $U$ test on skewed distribution. Additionally, the scale score differences between baseline and 1-year outcome assessment within each treatment group were analysed by using the paired $t$ test on normal distribution and Wilcoxon signed-rank test on skewed distribution. The CT findings were analysed using the Chisquare test.

\section{Results}

Out of our previous cohort [20], 14 patients were included who were treated with arthroscopic BMS in case of the presence of a fixable talar OCD. To create a similar and comparable cohort, we therefore only included the first 14 patients who were treated with arthroscopic LDDF. Both groups completed all questionnaires and the CT follow-up at 1 year postoperatively. The baseline characteristics are presented in Table 1. Patients in the LDFF group were significantly younger $(p<0.01)$ and had a lower body mass index (BMI) $(p<0.01)$. There was no significant difference in OCD classification or size of the lesion. Fixation was performed in nine cases with bio-compression screw(s), in three cases with chondral dart(s) and in two cases with a combination of both.

\section{Clinical results}

Both preoperatively and 1 year postoperatively, no significant differences in the AOFAS and NRS pain at rest and running were found between arthroscopic LDFF and arthroscopic BMS (Figs. 1, 2).

Within both treatment groups, the NRS pain and AOFAS improved significantly from preoperatively to 1 year postoperatively. After arthroscopic LDFF, the AOFAS significantly improved from 66 (SD 10.1) to 89 (SD 17.0) $(p=0.004)$. The NRS pain at rest significantly improved from 2.1 (SD 1.8) to 0.9 (SD 1.3) ( $p=0.043)$, and NRS pain when running improved from 7.4 (SD 1.9) to 2.5 (SD 3.1) ( $p=0.004)$ (Figs. 1, 2). After arthroscopic BMS, the AOFAS significantly improved from 57.1 (SD 13.6) to 83 (SD 15.9) $(p<0.001)$. The NRS pain at rest significantly improved from 3.3 (SD 1.5) to 0.9 (SD 1.7) $(p=0.001$ ), and NRS pain when running improved from 8.1 (SD 1.7) to 3.9 (SD 2.8) $(p<0.001)$ (Figs. 1, 2).

\section{Radiological results}

A significant difference $(p=0.02)$ was found in the healing of the subchondral bone plate between both groups. After arthroscopic BMS, a depressed subchondral bone plate was observed in 11 patients and three patients had a flush subchondral bone plate (Fig. 3), while after arthroscopic LDFF, a depressed subchondral bone plate was found

Table 1 Baseline characteristics of the patients

\begin{tabular}{lccl}
\hline & LDFF, $n=14$ & BMS, $n=14$ & $p$ value \\
\hline Age (years), median (IQR) & $17(16-18)$ & $23(20-30)$ & $<0.01$ \\
Gender, $n$ (\% male) & $5(36)$ & $5(36)$ & n.s. \\
BMI, mean (SD) & $22(3)$ & $27(4)$ & $<0.01$ \\
Included side, $n$ (\% right) & $10(71)$ & $12(86)$ & n.s. \\
OCD size, mean (SD) & & & \\
$\quad$ Anteroposterior (mm) & $13(2)$ & $12(3)$ & n.s. \\
Medial-lateral (mm) & $9(2)$ & $9(2)$ & n.s. \\
$\quad$ Superior-inferior (mm) & $6(3)$ & $5(2)$ & n.s. \\
OCD classification, $n(\%)$ & & & \\
$\quad$ Partially fractured & $2(14)$ & $2(14)$ & n.s. \\
$\quad$ Completely undisplaced & $12(86)$ & $10(72)$ & n.s. \\
$\quad$ fracture & & & \\
Displaced fracture & $0(0)$ & $2(14)$ & n.s. \\
\hline
\end{tabular}

$I Q R$ interquartile range, $S D$ standard deviation, n.s. not significant 


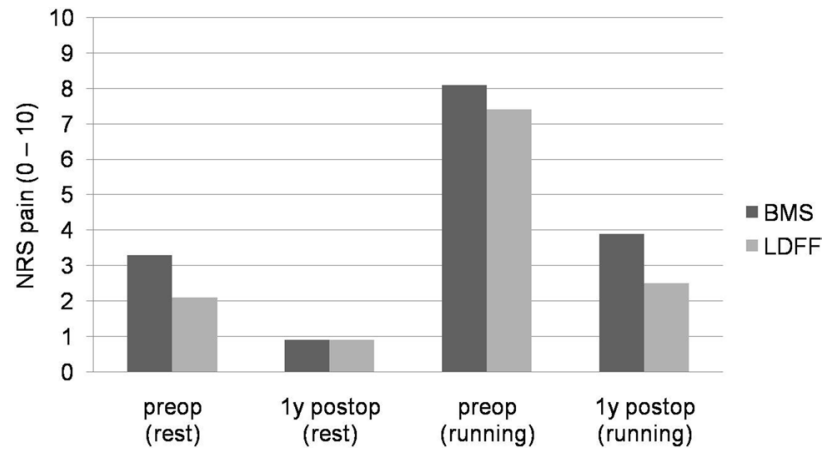

Fig. 1 Graph showing the mean numeric rating scales (NRSs) for pain (at rest and when running) pre- and postoperatively. No significant differences were found between arthroscopic LDFF and arthroscopic BMS

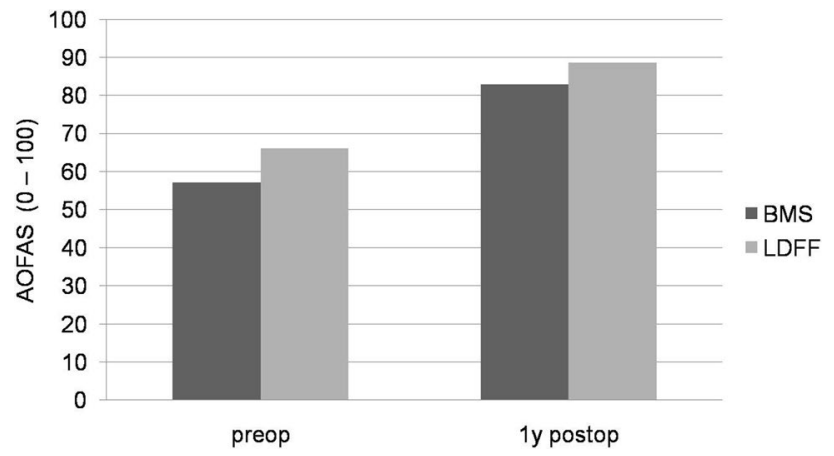

Fig. 2 Graph showing the mean American Orthopaedic Foot and Ankle Society (AOFAS) ankle-hindfoot score pre- and postoperatively. No significant differences were found between arthroscopic LDFF and arthroscopic BMS

in four patients and a flush subchondral bone plate in 10 patients (Fig. 4).

Union of the osteochondral fragment was found in nine patients after arthroscopic LDFF.

\section{Complications}

No serious adverse event occurred in either groups. One patient had prolonged wound leakage during the first week after arthroscopic BMS. No complications were reported after arthroscopic LDFF.

\section{Discussion}

The most important findings of the present comparative study were that no clinical differences were found between arthroscopic LDFF and arthroscopic BMS at 1-year followup. However, the subchondral bone plate restores significantly better after LDFF in comparison with BMS. Union
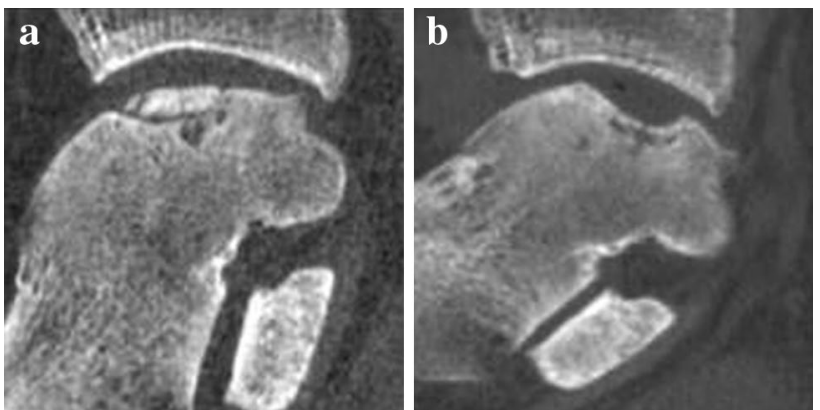

Fig. 3 a Preoperative sagittal CT of a medial osteochondral talar defect of a right ankle. b Postoperative sagittal CT of the same ankle after arthroscopic debridement and bone marrow stimulation (BMS) at 1-year follow-up
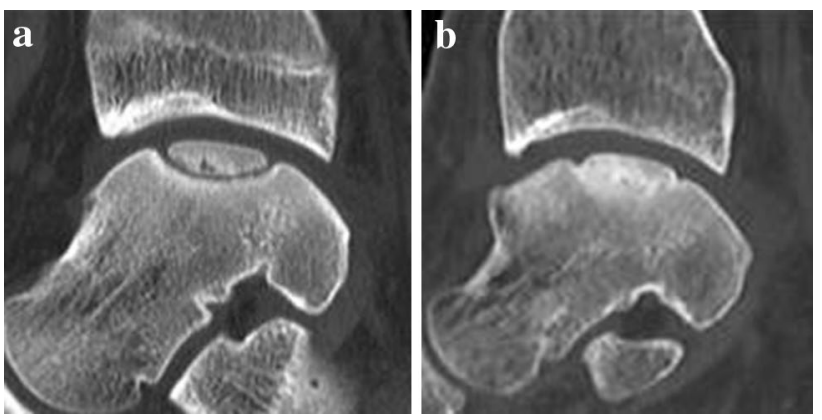

Fig. 4 a Preoperative sagittal $\mathrm{CT}$ of a medial osteochondral talar defect of a right ankle. b Postoperative sagittal CT of the same ankle after arthroscopic lift, drill, fill, and fix (LDFF) at 1-year follow-up

of the fragment was found in nine out of 14 patients, but was not associated with a better outcome. This could be explained because a non-united fragment was stabilized by scar tissue and was no longer an intra-articular loose body.

The healing of the subchondral bone plate is important in the surgical treatment of OCDs. Research has indicated that an irregular subchondral bone plate has a negative effect on cartilage repair and thus plays an important role in the development of osteoarthritis $[9,10,12,15,16]$. Progression of ankle osteoarthritis is seen in $33-34 \%$ of the patients following arthroscopic debridement and BMS at long-term follow-up [4, 16, 24]. Although the long-term clinical and radiological outcomes of the arthroscopic LDFF procedure have not been researched yet, it is postulated that progression of ankle osteoarthritis is less than in patients treated with BMS because the subchondral bone plate restores more in accordance with the normal congruency of the ankle. $78-100 \%$ of the patients were regarded clinically successful in case series describing open fixation of talar OCDs at mid-term follow-up [11, 18, 21]. Furthermore, in an earlier study we found no progression of osteoarthritis after open fixation of talar OCDs in children at mid-term follow-up [18]. 
To the best of our knowledge, this is the first prospective comparative study investigating the clinical and radiological changes between arthroscopic LDFF and arthroscopic BMS. Strengths of this study include the prospective methodology and the complete radiological and clinical follow-up. Furthermore, the defect size was equally distributed between both groups. This is important because larger defects are associated with poorer outcomes [2, 3, 17]. Limitations include the lack of longterm follow-up and power analysis. Furthermore, BMI was significantly lower and patients were significantly younger in the LDFF group. These factors are associated with superior outcomes $[18,20]$. However, it must be noted that none of the patients were classified as obese according to the WHO standards [29]. Furthermore, only skeletally mature patients were included in this study.

Based on the radiological results, fixation of a talar OCD with a bony fragment should be considered as the primary surgical treatment.

\section{Conclusion}

No clinical differences were found between arthroscopic debridement and BMS and arthroscopic LDFF in the treatment of osteochondral talar defects at 1-year followup. However, the subchondral bone plate restores significantly superior after arthroscopic LDFF compared to arthroscopic BMS.

\section{Compliance with ethical standards}

Conflict of interest The authors declare that they have no conflict of interest.

Funding No funding was received.

Ethical approval This study was approved by the local medical ethics committee at the University of Amsterdam with reference number MEC 08/326 and performed in accordance with the current ethical standards (Declaration of Helsinki).

Open Access This article is distributed under the terms of the Creative Commons Attribution 4.0 International License (http:// creativecommons.org/licenses/by/4.0/), which permits unrestricted use, distribution, and reproduction in any medium, provided you give appropriate credit to the original author(s) and the source, provide a link to the Creative Commons license, and indicate if changes were made.

\section{References}

1. Berndt AL, Harty M (1959) Transchondral fractures (osteochondritis dissecans) of the talus. J Bone Joint Surg Am 41:988-1020
2. Choi WJ, Park KK, Kim BS, Lee JW (2009) Osteochondral lesion of the talus: is there a critical defect size for poor outcome? Am J Sports Med 37:1974-1980

3. Chuckpaiwong B, Berkson EM, Theodore GH (2008) Microfracture for osteochondral lesions of the ankle: outcome analysis and outcome predictors of 105 cases. Arthroscopy 24:106-112

4. Ferkel RD, Zanotti RM, Komenda GA, Sgaglione NA, Cheng MS, Applegate GR et al (2008) Arthroscopic treatment of chronic osteochondral lesions of the talus: long-term results. Am J Sports Med 36:1750-1762

5. Gagliese L, Weizblit N, Ellis W, Chan VW (2005) The measurement of postoperative pain: a comparison of intensity scales in younger and older surgical patients. Pain 117:412-420

6. Ibrahim T, Beiri A, Azzabi M, Best AJ, Taylor GJ, Menon DK (2007) Reliability and validity of the subjective component of the American Orthopaedic Foot and Ankle Society clinical rating scales. J Foot Ankle Surg 46:65-74

7. Kerkhoffs GM, Reilingh ML, Gerards RM, de Leeuw PA (2016) Lift, drill, fill and fix (LDFF): a new arthroscopic treatment for talar osteochondral defects. Knee Surg Sports Traumatol Arthrosc 24:1265-1271

8. Kitaoka HB, Alexander IJ, Adelaar RS, Nunley JA, Myerson MS, Sanders M (1994) Clinical rating systems for the ankle-hindfoot, midfoot, hallux, and lesser toes. Foot Ankle Int 15:349-353

9. Koulalis D, Schultz W, Heyden M (2002) Autologous chondrocyte transplantation for osteochondritis dissecans of the talus. Clin Orthop Relat Res 395:186-192

10. Koulalis D, Schultz W, Heyden M, Konig F (2004) Autologous osteochondral grafts in the treatment of cartilage defects of the knee joint. Knee Surg Sports Traumatol Arthrosc 12:329-334

11. Kumai T, Takakura Y, Kitada C, Tanaka Y, Hayashi K (2002) Fixation of osteochondral lesions of the talus using cortical bone pegs. J Bone Joint Surg Br 84:369-374

12. Lynn AK, Brooks RA, Bonfield W, Rushton N (2004) Repair of defects in articular joints. Prospects for material-based solutions in tissue engineering. J Bone Joint Surg Br 86:1093-1099

13. Marsh JL, Buckwalter J, Gelberman R, Dirschl D, Olson S, Brown $T$ et al (2002) Articular fractures: does an anatomic reduction really change the result? J Bone Joint Surg Am 84:1259-1271

14. Nakasa T, Adachi N, Kato T, Ochi M (2014) Appearance of subchondral bone in computed tomography is related to cartilage damage in osteochondral lesions of the talar dome. Foot Ankle Int 35:600-606

15. Orth P, Cucchiarini M, Kaul G, Ong MF, Graber S, Kohn DM et al (2012) Temporal and spatial migration pattern of the subchondral bone plate in a rabbit osteochondral defect model. Osteoarthritis Cartilage 20:1161-1169

16. Qiu YS, Shahgaldi BF, Revell WJ, Heatley FW (2003) Observations of subchondral plate advancement during osteochondral repair: a histomorphometric and mechanical study in the rabbit femoral condyle. Osteoarthritis Cartilage 11:810-820

17. Ramponi L, Yasui Y, Murawski CD, Ferkel RD, DiGiovanni CW, Kerkhoffs GM et al (2016) Lesion size is a predictor of clinical outcomes after bone marrow stimulation for osteochondral lesions of the talus: a systematic review. Am J Sports Med. doi:10.1177/0363546516668292

18. Reilingh ML, Kerkhoffs GM, Telkamp CJ, Struijs PA, van Dijk CN (2014) Treatment of osteochondral defects of the talus in children. Knee Surg Sports Traumatol Arthrosc 22:2243-2249

19. Reilingh ML, van Bergen CJ, Blankevoort L, Gerards RM, van Eekeren IC, Kerkhoffs GM et al (2016) Computed tomography analysis of osteochondral defects of the talus after arthroscopic debridement and microfracture. Knee Surg Sports Traumatol Arthrosc 24:1286-1292

20. Reilingh ML, van Bergen CJ, Gerards RM, van Eekeren IC, de Haan RJ, Sierevelt IN et al (2016) Effects of pulsed 
electromagnetic fields on return to sports after arthroscopic debridement and microfracture of osteochondral talar defects: a randomized, double-blind, placebo-controlled, multicenter trial. Am J Sports Med 44:1292-1300

21. Schuh A, Salminen S, Zeiler G, Schraml A (2004) Results of fixation of osteochondral lesions of the talus using K-wires [German]. Zentralbl Chir 129:470-475

22. Scranton PE Jr, Frey CC, Feder KS (2006) Outcome of osteochondral autograft transplantation for type-V cystic osteochondral lesions of the talus. J Bone Joint Surg Br 88:614-619

23. Stufkens SA, Knupp M, Horisberger M, Lampert C, Hintermann B (2010) Cartilage lesions and the development of osteoarthritis after internal fixation of ankle fractures: a prospective study. J Bone Joint Surg Am 92:279-286

24. van Bergen CJ, Kox LS, Maas M, Sierevelt IN, Kerkhoffs GM, van Dijk CN (2013) Arthroscopic treatment of osteochondral defects of the talus: outcomes at eight to twenty years of followup. J Bone Joint Surg Am 95:519-525

25. van Dijk CN, Reilingh ML, Zengerink M, van Bergen CJ (2010) Osteochondral defects in the ankle: why painful? Knee Surg Sports Traumatol Arthrosc 18:570-580
26. van Dijk CN, Verhagen RA, Tol JL (1997) Arthroscopy for problems after ankle fracture. J Bone Joint Surg Br 79:280-284

27. van Eekeren IC, Reilingh ML, van Dijk CN (2012) Rehabilitation and return-to-sports activity after debridement and bone marrow stimulation of osteochondral talar defects. Sports Med 42:857-870

28. Verhagen RA, Maas M, Dijkgraaf MG, Tol JL, Krips R, van Dijk CN (2005) Prospective study on diagnostic strategies in osteochondral lesions of the talus. Is MRI superior to helical CT? J Bone Joint Surg Br 87:41-46

29. World Health Organisation (2006) Obesity and overweight. www.who.int/mediacentre/factsheets/fs311/en/index.html

30. Zengerink M, Struijs PA, Tol JL, van Dijk CN (2010) Treatment of osteochondral lesions of the talus: a systematic review. Knee Surg Sports Traumatol Arthrosc 18:238-246

31. Zengerink M, van Dijk CN (2012) Complications in ankle arthroscopy. Knee Surg Sports Traumatol Arthrosc 20:1420-1431

32. Zinman C, Wolfson N, Reis ND (1988) Osteochondritis dissecans of the dome of the talus. Computed tomography scanning in diagnosis and follow-up. J Bone Joint Surg Am 70:1017-1019 